\title{
A Cool-Handed Patient with a Right Hemispheric Stroke
}

\author{
Glen Jicking, James Scozzafava, Mickael Mouradian, Randy MacDonald, Ashfaq Shuaib
}

Can. J. Neurol. Sci. 2008; 35: 655-657

A 51-year-old right-handed female awoke with weakness of her left face, arm and leg, in addition to a right gaze preference and dysarthria. In the emergency department she was diagnosed with a right middle cerebral artery stroke and the stroke team was consulted. Her right hand and forearm was also noted to be cool, purple and painful to touch. Although she had a two-day history of nausea, emesis and diarrhea; she denied any trauma, shortness of breath or pain in her back, chest or neck. She was previously healthy with no history of arterial or venous thromboembolism including deep venous thrombosis, pulmonary embolism or miscarriage. No vascular risk factors were identified and her family history was unremarkable.

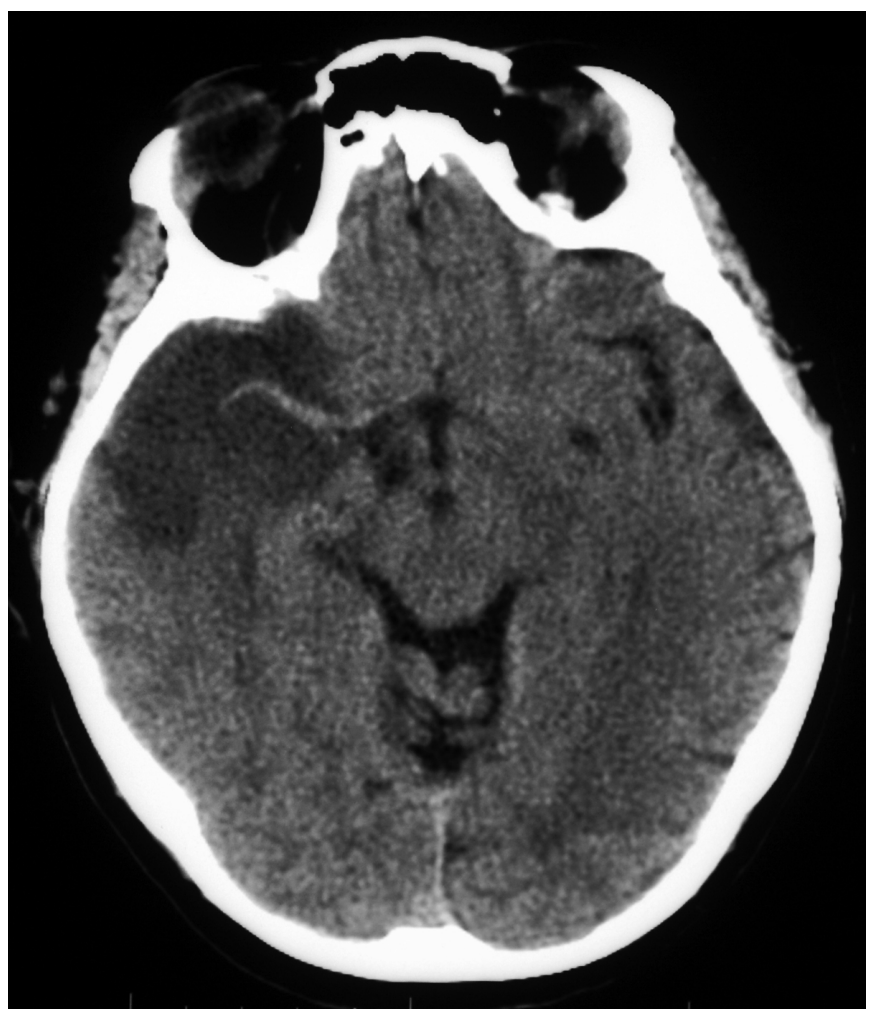

Figure 1: Axial non-contrast CT head showing subacute right middle cerebral artery stroke, with associated hyperdense right middle cerebral artery.
On examination, her blood pressure was $175 / 80 \mathrm{mmHg}$ in the right arm and 190/85 $\mathrm{mmHg}$ in the left. Right ulnar and radial pulses were not palpable at the wrist or elbow. The right hand was tensely swollen, discoloured, cool and tender to palpation. On cranial nerve examination, there was no ptosis or miosis. There was a left gaze palsy, and a left facial droop. Motor examination revealed a dense hemiplegia on the left side. Reflexes were brisk on the left and her left toe was upgoing. The remainder of her neurological examination was unremarkable.

Routine investigations were normal, including hemoglobin, platelets, international normalized ratio (INR), partial thromboplastin time (PTT), chest xray, and electrocardiogram. Computed tomography (CT) of the brain showed a recent large right middle cerebral artery stroke with a dense middle cerebral artery sign (Figure 1). Concern regarding the right hand findings led to an urgent angiogram of the right arm. A large $1.5 \mathrm{~cm}$ mobile mass was identified in the brachiocephalic artery. Furthermore, there was reduced flow to the right subclavian artery and the right common carotid artery extending into the bifurcation of the internal and external carotid arteries (Figure 2).

The patient underwent an urgent surgical embolectomy of the brachiocephalic artery, the right brachial artery and the right carotid artery. Distal control was achieved by introducing a Fogarty catheter distal to the carotid thrombus via the right internal carotid artery. It was advanced proximally toward the aorta, and the thrombus was extracted. Thrombus was also extracted from the brachial artery by introducing a Fogarty catheter just above the level of the birfurcation of the brachial artery. The thrombus was sent for pathologic evaluation and no evidence of malignancy or infection was identified. The patient did not experience any complications from the procedure. The patient was started on anticoagulation following the procedure. Despite being on coumadin with an INR of 2.6, the patient developed recurrent right arm ischemia six days later and required repeat right brachial artery embolectomy with the

From the Division of Neurology (GJ, MM, AS), Department of Emergency Medicine (RM), University of Alberta, Edmonton; Department of Critical Care Medicine (JS), University of Calgary, Calgary, Alberta, Canada.

Received December 15, 2007. Final Revisions Submitted May 9, 2008. Correspondence to: James Scozzafava, Department of Critical Care Medicine, Foothills Medical Centre, 1403-29th Street NW, Calgary, Alberta, T2N 2T9, Canada. 
addition of $5 \mathrm{mg}$ tissue plasminogen activator (tPA) in each of the distal ulnar and radial arteries. During surgery, the patient was noted to form clot very readily despite a therapeutic INR. She tolerated the procedure well.

A work up for a hypercoagulable state was negative including antiphospholipid antibodies, hyperhomocystenemia, prothrombin gene mutation, antithrombin III deficiency, factor $\mathrm{V}$ Leiden, factor VIII deficiency, factor IX deficiency, factor XI deficiency, fibrinogen, protein $\mathrm{C}$ deficiency and protein $\mathrm{S}$ deficiency. A transesophageal echo (TEE) revealed a small patent foramen ovale (PFO) with no atrial septal aneurysm. No evidence of venous thrombosis was identified on lower extremity ultrasound or CT chest. A screen for malignancy was negative, including CT chest-abdomen-pelvis, abdominal ultrasound and carcino-embryonic antigen (CEA) levels. The patient was discharged home on coumadin and aspirin. She had full function of her right upper extremity within two weeks of the interventional therapies. Her left sided hemiplegia showed gradual improvement with rehabilitation.

\section{Discussion}

The initial assessment of stroke patients is often the most critical period with regards to urgent investigation and potential treatment. In stroke patients there are often associated medical conditions that can be identified, particularly in the young stroke patient. A patient presenting with contralateral hemiplegia with an ipsilateral cool, pulseless hand suggests a process affecting multiple vascular territories. Diagnostic considerations include a proximal vascular lesion such as an aortic dissection and a pseudoaneurysm of the brachiocephalic artery. An aortic dissection must be considered in any patient presenting with asymmetry of peripheral blood pressures and/or pulses and although aortic dissection classically presents with sharp chest or abdominal pain, there are reports of painless aortic dissection, presenting only with neurologic dysfunction and a widened mediastinum on chest $\mathrm{x}$-ray. ${ }^{1}$ Pseudoaneurysm of the brachiocephalic artery is an uncommon vascular lesion related to trauma or first rib impingement. It has been reported to cause left hemiplegia and right upper extremity ischemia. ${ }^{2-4}$ Our patient had no clinical or radiographic evidence of an aortic dissection or a brachiocephalic pseudoaneurysm.

Cardiac emboli to multiple vascular territories is an important consideration and sources of cardiac arterial emboli include cardiac arrhythmias such as atrial fibrillation and atrial flutter, valvular lesions seen in endocarditis, and cardiac muscle lesions such as acute anterior myocardial infarction. A defect in the cardiac septum such as a large PFO, can cause a paradoxical embolism of venous thrombus into the arterial system and a case report with a large PFO has described a similar presentation. ${ }^{5}$ Our patient had a small PFO with no evidence of venous thrombosis, and no other identified cardioembolic source.

Certain hypercoagulable states can also cause arterial thrombosis in multiple territories, including antiphospholipid antibodies, hyperhomocystenemia, and paroxysmal nocturnal hemoglobinuria. Malignancy can also cause a hypercoagulable state through a low grade disseminated intravascular coagulopathy which shifts the coagulation cascade towards clotting. The hypercoagulable state can lead to thrombus formation throughout the vascular system, particularly involving the heart

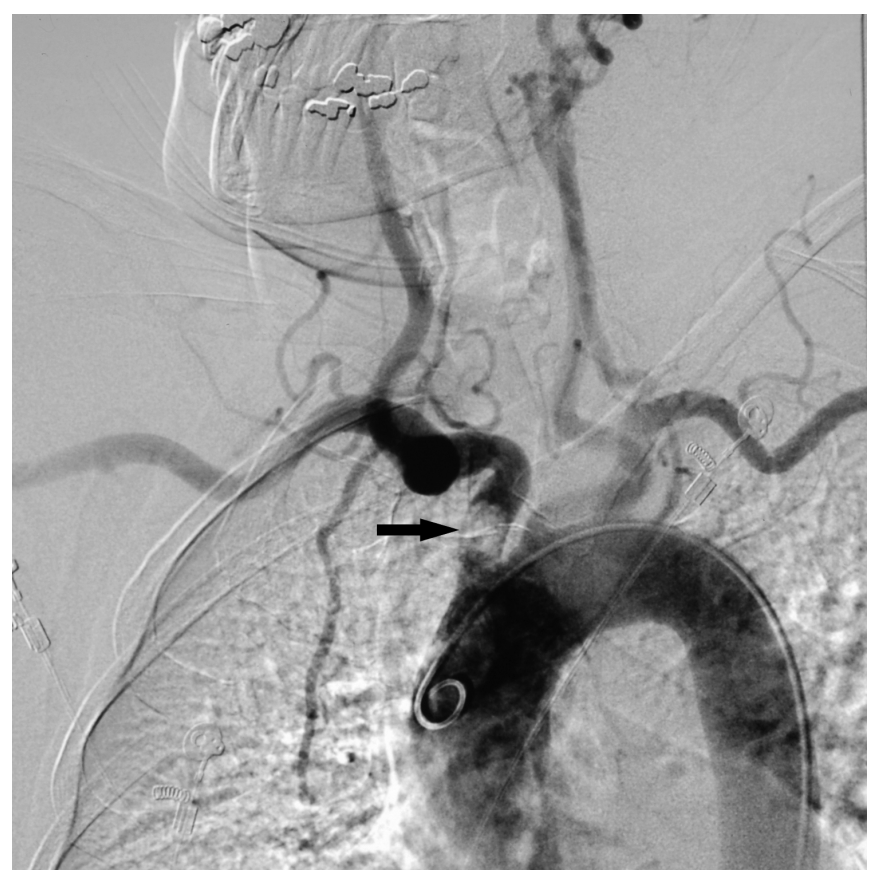

Figure 2: Arch aortogram showing a $1.5 \mathrm{~cm}$ filling defect of the proximal brachiocephalic trunk that appears mobile. Distal branches showed delayed filling. The left common carotid, subclavian and vertebral arteries appeared normal. The left vertebral artery was dominant.

valves. Embolic strokes can result from a nonbacterial thrombotic endocarditis and should be considered in any young patient with multiple territory strokes. ${ }^{6}$ In our patient, a hypercoaguable state was strongly suspected based on the tendency to form thrombus during surgery and her recurrent thrombosis despite anticoagulation. However, investigations did not identify a hypercoagulable disorder and a detailed search for a possible malignancy was negative. Furthermore, TEE did not reveal a valvular abnormality.

Stroke associated with thrombus in the brachiocephalic artery is rare and consequently its management is unclear. Anticoagulation to prevent thrombus formation carries a significant risk of hemorrhagic transformation of the stroke, particularly in large middle cerebral artery strokes within the first week of the stroke. Interventional angiographic procedures, including mechanical embolectomy and intra-arterial thrombolytic therapy represent an alternate option. Interventional angiographic procedures often involve a proximal approach to the thrombus and inadequate distal control can result in increased risk of distal embolisation. Although distal emboli protection devices are available, these have to be passed through the clot first and can precipitate distal embolisation as well.

A distal surgical approach is possible in some circumstances, however also carries the risk of precipitating embolic events, particularly through retrograde and collateral circulation. In our patient, concern centered around the possibility of further embolic strokes resulting from an interventional procedure, 
either by propagating the right carotid artery thrombus distally into the right internal carotid artery and middle cerebral artery or by precipitating a retrograde embolism from the right common carotid artery to the right vertebral artery. Surgical embolectomy was successful and did not result in further embolic events in our patient. This may have been due to both good distal control during the procedure and a dominant left vertebral artery, minimizing the risk of a retrograde embolism.

In our patient, the most important aspect of her investigation and management involved the early and accurate examination. Stroke patients often present to the hospital with coexisting medical conditions. Careful assessment is required to identify medical conditions such as aortic dissection, carotid dissection, myocardial infarction and cardiac dysrhythmia. Careful assessment is also required to identify other acute medical issues that may require urgent investigation and management, such as other tissue ischemia. In our young patient early detection of critical examination findings led to surgical intervention that effectively saved her ischemic right arm and prevented bilateral upper extremity functional impairment.

\section{REFERENCES}

1. Morita S, Shibata M, Nakagawa Y, Yamamoto I, Inokuchi S. Painless acute aortic dissection with a left hemiparesis. Neurocritical Care. 2006;4(3):234-6.

2. Hoobler SW. The syndrome of cervical rib with subclavian arterial thrombosis and hemiplegia due to cerebral embolism: a case report. N Engl J Med. 1942;226:942-4.

3. Fields WS, Lemak NA, Ben-Menachem Y. Thoracic outlet syndrome: review and reference to stroke in a major league pitcher. AJR Am J Roentgenol. 1986;146:809-14.

4. Al-Hassan HK, Sattar MA, Eklof B. Embolic brain infarction: a rare complication of thoracic outlet syndrome-a report of two cases. J Cardiovasc Surg. 1988;29:322-5.

5. Turnbull RG, Tsang V, Teal P, Salvian A. Successful innominate thromboembolectomy of a paradoxic embolus. J Vasc Surg. 1998;28:742-5.

6. Scozzafava J, Hussain MS, Ahmed SN, Khan K. Recurrent strokes in a 46-year-old woman: rapidly progressive nonbacterial thrombotic endocarditis. CMAJ. 2006;175(9):1055-6. 